Article

\title{
Performance Evaluation of Energy Transition Based on the Technique for Order Preference by a Similar to Ideal Solution and Support Vector Machine Optimized by an Improved Artificial Bee Colony Algorithm
}

\author{
Zhen $\mathrm{Li}^{1}$, Yun $\mathrm{Li}^{2}{ }^{2}$ * and Yanbin $\mathrm{Li}^{1}$ \\ 1 School of Economics and Management, North China Electric Power University, Beijing 102206, China \\ 2 China National Institute of Standardization, Beijing 100191, China \\ * Correspondence: liyun1@cnis.ac.cn; Tel.: +86-15210168026
}

Received: 25 June 2019; Accepted: 5 August 2019; Published: 8 August 2019

check for updates

\begin{abstract}
Energy transition is an important factor when dealing with climate change and energy crisis under resource constraints. The performance evaluation of it is significant for improving and promoting the process of energy transition. This paper explores the application of the support vector machine improved by the artificial bee colony algorithm (IABC-SVM) method in the energy transition performance evaluation process. It provides an intelligent evaluation tool for the evaluation of the regional energy transition performance. Firstly, the evaluation indicator system of energy transition is constructed from five dimensions: energy supply, demand, efficiency, institution, and environment. Then, the technique for order preference by a similar to ideal solution improved by a combination weighting (CW-TOPSIS) method and IABC-SVM are constructed. After that, according to the evaluation values of 30 provinces in China calculated by CW-TOPSIS, 10 -fold cross validation is used to compare the errors of support vector machine (SVM), support vector machine optimized by the artificial bee colony algorithm (ABC-SVM), and IABC-SVM, which proves the effectiveness and accuracy of IABC-SVM in evaluating the performance of energy transition. Finally, the IABC-SVM is used to evaluate the energy transition performance of 30 provinces in 2016. Through a comparative analysis, the relevant suggestions of energy transition are put forward.
\end{abstract}

Keywords: energy transition; evaluation; the technique for order preference by a similar to ideal solution improved by combination weighting; support vector machine improved by the artificial bee colony algorithm

\section{Introduction}

With the increasing attention that is being paid to the issues of energy security, climate change, and ecological environment protection by the international community, accelerating energy transition has become a universal consensus and concerted action of all countries in the world [1]. In 2014, China also put forward the energy transition strategy of four revolutions, one cooperation, aimed at building a clean, low-carbon, safe, and efficient modern energy system [2]. The evaluation of energy transition can not only clearly recognize the performance of regional energy transformation, but also guide the actual work of energy transition and development, identify problems in a timely manner, and adjust the development path. It is of great significance to grasp the process of energy transition and promote the energy revolution.

The performance evaluation of energy transition is a complex and multi-indicator comprehensive evaluation problem. The validity of the evaluation depends on the rationality of the indicator system 
and the scientificity of the evaluation method. In recent years, scholars have studied many aspects of energy evaluation, such as energy development evaluation [3-5], energy security evaluation [6-10], energy structure evaluation [11-14], energy efficiency evaluation [15-18], etc.

The evaluation of energy development can also be divided into the evaluation of sustainable energy development, the evaluation and prediction of energy resources, and the evaluation of environmental benefits of energy development. Reference [3] combined the energy demand with the growth rates in various sectors of the economy, formatting standardized data sets, which are used separately by member states of the European community for multi-regional and multi-cycle energy analysis. Li et al. [4] constructed the comprehensive evaluation index of renewable energy from the four aspects of economy, technology, resources, and the environment, and the model of the comprehensive evaluation and structure optimization of renewable energy in China was established. Su et al. [5] evaluated the impact of energy pollution in the whole supply chain from production and transportation to consumer terminals from the perspective of the product life cycle.

For the evaluation of energy security, Cherp et al. [6] argued that climate policy can reduce the dependence of the energy supply, energy structure, and energy trade on fossil energy consumption and economic growth. The composition of the energy security indicator system is discussed in detail in reference [7], which considers the indicators for measuring energy security, including predictive indicators and outcome indicators. Blum and Legey [8] analyzed the factors affecting energy security from both a supply and demand perspective, and pointed out that an economy should be able to provide sufficient, affordable, and environmentally sustainable energy in order to maintain maximum prosperity. Sovacool et al. [9] constructed the energy security evaluation index system from five aspects: availability, affordability, technology, sustainability, and regularity, and evaluated the energy security performance of 18 countries. Reference [10] took Thailand as an example, and three energy scenarios were established to assess the future energy security from the energy demand, diversified resources, environment, and market.

For the evaluation of energy structure, reference [11] introduced the classical control theory to analyze the change of the primary energy structure more scientifically based on the energy-economy model. Fan et al. [12] conducted an empirical analysis of the rationality of the energy structure from the three dimensions of the environment, social economy, and energy development, and found that the rationality of the energy structure presents a continuous fluctuation state. Chandler [13] explored the diversity of energy consumption in the U.S. electricity sector from the perspective of sustainable development. Reference [14] evaluated the low-carbon energy consumption structure in the UK through a comprehensive macroeconomic model.

For the evaluation of energy efficiency, a comprehensive energy efficiency evaluation index system, including three subsystems of $3 \mathrm{E}$ (energy-economy-environment), is constructed in reference [15]. Reference [16] took energy, capital, labor, and other factors into account, and the Data Envelopment Analysis (DEA) model was used to measure the efficiency of total factor productivity. Herrerias [17] calculated the energy efficiency of 83 countries using the weighted dynamic distribution method. Cluster analysis was used to study the difference in the energy intensity of quality inspection in different countries by the Theil index in reference [18].

However, there is little research on the performance evaluation of energy transition. Zhu and Shi [19] proposed the index system of environmental performance evaluation of energy transition policy, and the comprehensive scoring method was used to evaluate the environmental performance of 2010 and 2015. Gu and Zhang [1] divided energy transition into technology transition and system transition, and the index analysis method was used to measure the degree of energy transition in China, the United States, and Germany.

As for evaluation methods, the analytic network process (ANP) [20], fuzzy comprehensive evaluation method [21], data envelopment analysis (DEA) [22], matter element extension method [23], and TOPSIS [24] are widely used in evaluation. However, for complex evaluation problems, the knowledge of experts can be obtained through an intelligence algorithm from traditional evaluation 
methods, and a non-linear mapping relationship from attribute values of evaluation indicators to output evaluation can be established. When evaluating other similar problems, only indicator data of the object to be evaluated is input, and the trained intelligent evaluation model can then evaluate automatically and quickly. Reference [25] explored the application of back propagation (BP) artificial neural network in building energy demand side management. Niu et al. [26] introduced the application of the least square support vector machine improved by a modified fly optimization algorithm model in evaluating the sustainability of power grid construction projects. Niu et al. [27] introduced the application of the least square support vector machine improved by the ant colony algorithm model in evaluating the operating efficiency of electricity sales companies.

To sum up, there are few studies on the performance evaluation of energy transition. This paper combines the objective of energy transition, and constructs an evaluation indicator system from energy supply, demand, efficiency, institution, and environmental dimensions.

In addition, the qualitative indicator score and empowerment are involved in the complex expert scoring process in traditional evaluation methods. Therefore, making full use of the advantages of big data and intelligent algorithms, the application of an intelligent evaluation method in energy transition performance evaluation is explored, which makes the evaluation more scientific, efficient, economical, and practical.

The contribution of this paper lies in the combination of traditional methods and intelligent algorithms, and the performance evaluation of energy transformation through the IABC-SVM model, which provides an intelligent tool for an evaluation of the regional energy transition performance. The IABC-SVM evaluation model has the advantages of accuracy and simplicity. The trained model can be used to evaluate the future energy transition situation reasonably and effectively. The innovations of this article are as follows:

1. The construction of the indicator system. Considering the optimization of the energy structure, technological progress, market-oriented reform process, energy saving, and emission reduction, this paper establishes an indicator system from five aspects: energy supply, demand, efficiency, institution, and the environment, to evaluate the performance of regional energy transition;

2. The construction of the evaluation model. This paper evaluates the energy transition performance of 30 provinces in China by IABC-SVM, which is an innovative exploration and attempt of an intelligent algorithm in the field of energy. The structure of IABC-SVM is relatively simple, and has a good generalization ability. When evaluating the performance of energy transition, it can improve the accuracy of judgment and provide more accurate decision support.

The remainder of this study is organized as shown as Figure 1. Section 2 constructs an indicators system of the energy transition performance. Section 3 presents the methodology adopted in this paper, including the TOPSIS method and IABC-SVM model. The validity of the IABC-SVM model is proved by case analysis, and the energy transition situation of 30 provinces in China in 2015 and 2016 is compared and analyzed in Section 4. Finally, we summarize the conclusions of this paper in Section 5. 


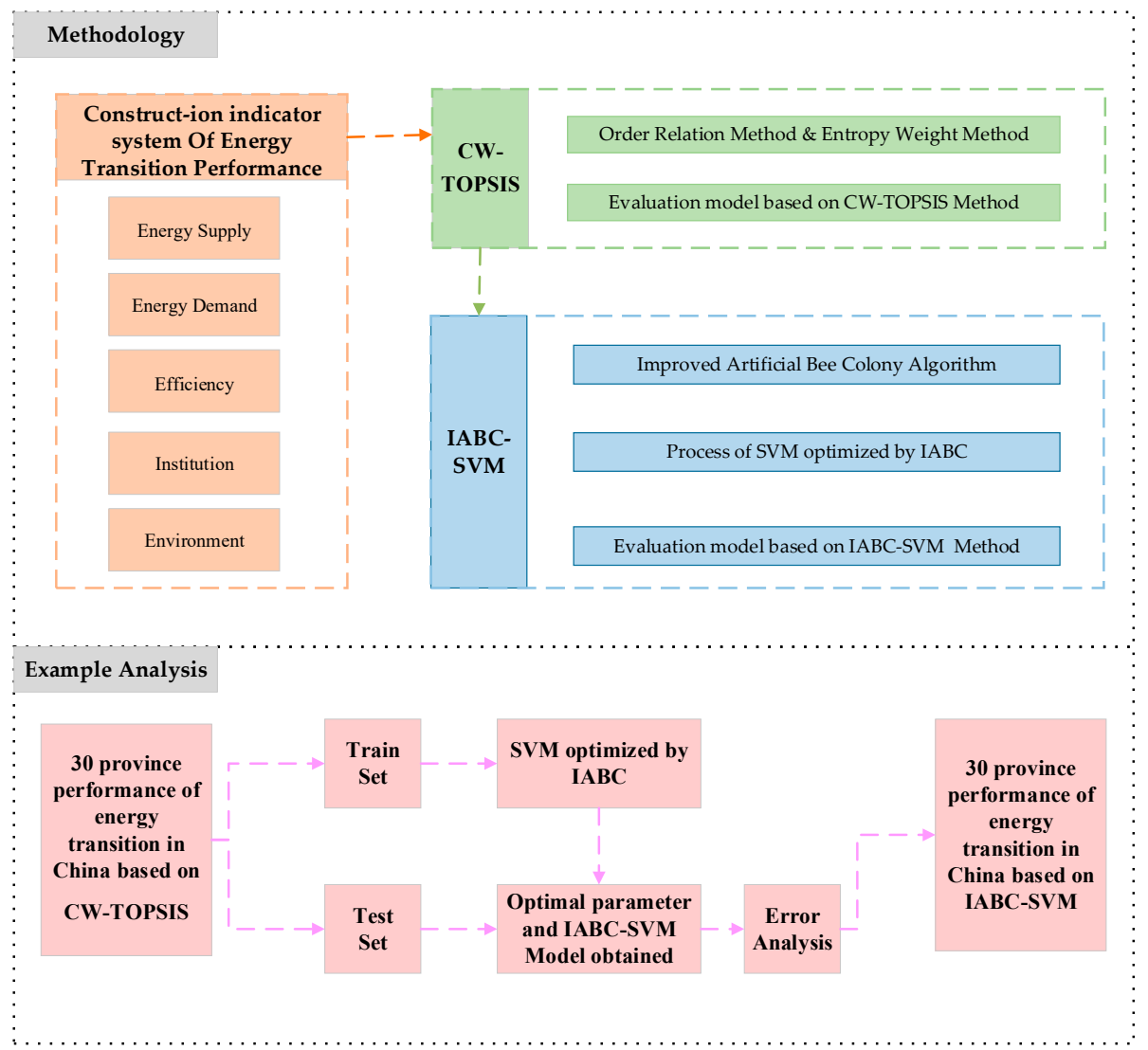

Figure 1. The structure of this study.

\section{The Indicator System for a Performance Evaluation of Energy Transition}

Energy transition aims to achieve the structural optimization of energy, efficiency improvement, and coordinated development with the economy and environment. Therefore, the indicator system of energy transition is mainly used to evaluate the transition performance of the energy supply, demand, efficiency, institution, and environment.

\subsection{Construction of the Evaluation Indicator System}

Through a large literature survey and expert consultation, five first-level indicators and eighteen second-level indicators have been constructed from the dimensions of supply, demand, efficiency, institution, and the environment, shown as Figure 2. According to the evaluation indicator system, the degree of energy transition in different regions can be determined. At the same time, we can also understand the transformation of different aspects from five dimensions, which can clearly reveal the focus of further transformation, and has guiding significance for choosing a more appropriate energy transition path. 


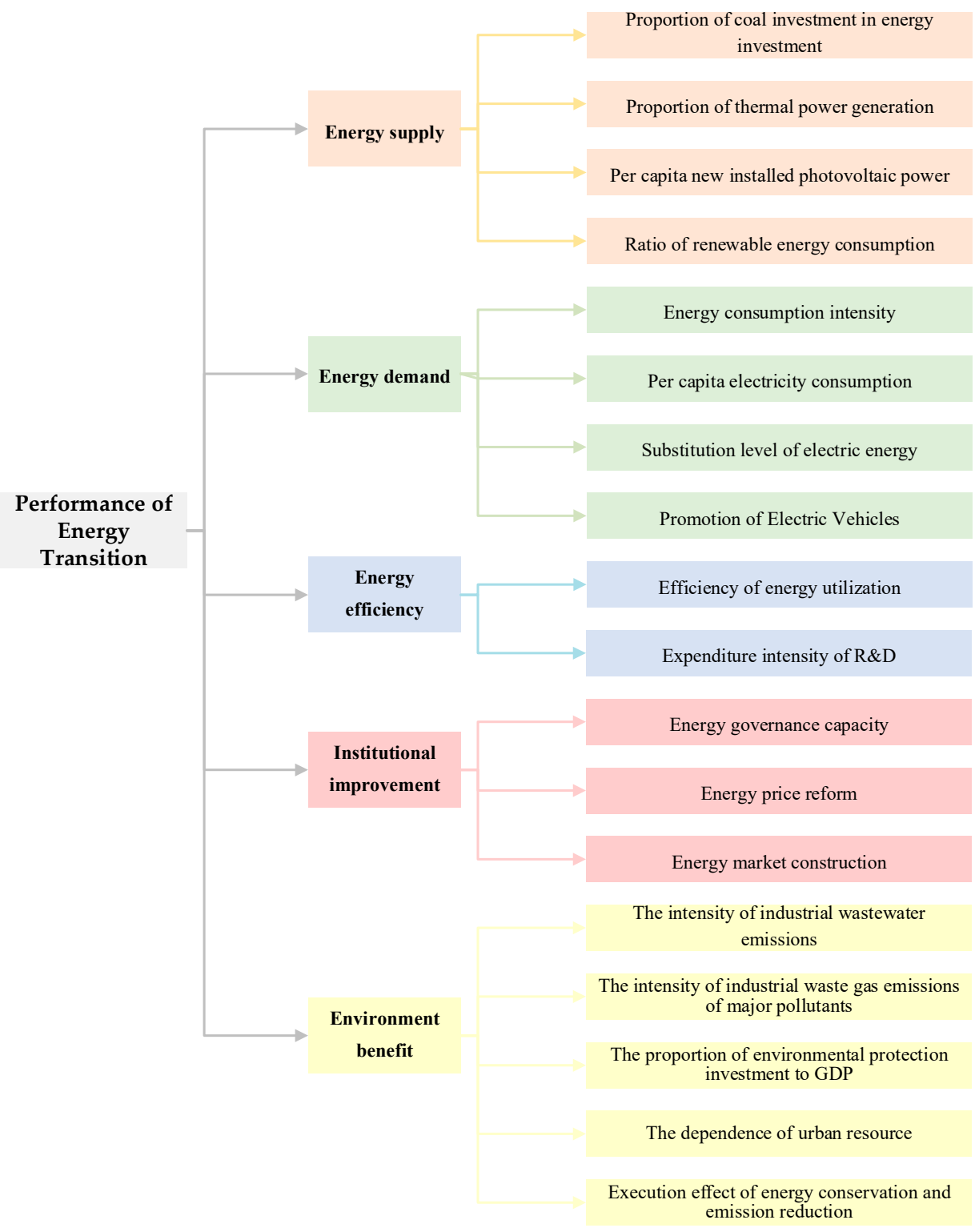

Figure 2. The indicator system used for the performance evaluation of energy transition.

\subsection{Indicator Description}

\section{(1) Energy supply}

Energy supply consists of four quantitative indicators: (1) Proportion of coal investment in energy investment equals investment in the coal mining and processing industry divided by investment in the energy industry, which can reflect the structural effect of energy supply [28]; (2) proportion of thermal power generation equals thermal power generation divided by power generation, which can reflect the performance of energy structure adjustment [29]; (3) per capita new installed photovoltaic power equals new installed capacity of photovoltaic power divided by the population, which reflects the importance of new energy sources in the reform of the energy supply side in a region [30]; (4) ratio of renewable energy consumption. Improving the proportion of non-fossil energy consumption, such as water, wind, and light, has become an important guarantee for the sustainable energy development of China.

(2) Energy demand

Energy demand consists of four indicators: (1) Energy consumption intensity refers to the level of energy consumption per unit of national economy in production. The change of this indicator is 
influenced by the technical factors of total energy consumption, which can reflect the structure of energy demand to a certain extent [31]; (2) per capita electricity consumption equals electricity consumption divided by the population, which can accurately reflect the internal relationship between energy consumption and economic growth; (3) substitution level of electric energy. From the point of view of the energy use form and policy trend, improving the level of electric energy substitution will be the main trend of energy development in the future; (4) promotion of electric vehicles. The popularization of electric vehicles can not only solve the problem of the shortage of petroleum reserves, but also solve the problem of environmental pollution to a certain extent.

(3) Energy efficiency

The improvement of energy efficiency is the result of the improvement of production technology and management level in the final analysis. Energy efficiency consists of two indicators: (1) Efficiency of energy utilization refers to the amount of economic output that a certain amount of energy input can bring, which measures support of energy for output as a factor of production; (2) expenditure intensity of research and development $(R \& D)$ equals $R \& D$ funds of industrial enterprises above the scale divided by the gross domestic product (GDP), which can measure the importance of regional technological innovation [32].

(4) Institutional improvement

Institutional improvement consists of three qualitative indicators, including the energy governance capacity, energy price reform, and energy market construction. According to the reform of energy areas and key links in each province, the process of energy system reform is evaluated. The scoring basis should include, but is not limited to, the construction of the ancillary service market, renewable energy quota system, green power certificate trading system, energy futures market, new energy subsidies, energy industry standards, etc.

(5) Environmental benefit

Environmental benefits are an important dimension when evaluating the performance of energy transition. Considering the availability of data, we select three quantitative indicators and two qualitative indicators from the perspective of the relationship between consumption lifestyles and the emission or resource extractions in a given area [33]. The intensity of industrial waste water emissions and the intensity of industrial waste gas emissions of major pollutants can measure the cleanness of energy consumption in different regions to some extent. The proportion of environmental protection investment in GDP equals the investment in industrial pollution control divided by the GDP, which can reflect the enthusiasm of a region in relation to pollution control and the degree of industrial transformation and upgrading [34]. The dependence of urban resources, the execution effect of energy conservation, and emission reduction is graded comprehensively by experts according to the policies of various regions, including policies on energy development and distribution, energy structure optimization and adjustment, the clean and efficient use of coal, and other policies, as well as regional energy strategies and environmental governance action plans, regional comprehensive energy development plans, and special plans.

In view of the differences in the dimension of the energy transition evaluation data and the different trends of the impact on the energy transition performance, it is necessary to standardize the indicator before comprehensive evaluation. The benefit indicator and cost indicator are standardized according to Formulas (1) and (2), respectively.

$$
\begin{aligned}
x_{i j}^{*} & =\frac{x_{i j}-\max x_{j}}{\max x_{j}-\min x_{j}} \\
x_{i j}^{*} & =\frac{\max x_{j}-x_{i j}}{\max x_{j}-\min x_{j}}
\end{aligned}
$$




\section{Methodology}

In this section, the TOPSIS evaluation model improved by a combination weighting method is introduced, and the SVM model optimized by the improved artificial bee colony algorithm algorithm is then introduced. Finally, the combination of the traditional evaluation model and intelligent evaluation model is introduced.

3.1. Evaluation Model Based on the Technique for Order Preference by a Similar to Ideal Solution Improved by a Combination Weighting Method

3.1.1. Combination Weighting Method with an Order Relation Method and Entropy Weight Method

(1) The subjective weight determined by the order relation

The order relation method is a subjective weighting method which does not need a consistency test. It is a subjective weighting method that first ranks the evaluation indexes qualitatively, then judges the adjacent indexes rationally (importance ratio), and finally calculates quantitatively. The advantage of the order relation method is that it does not need to construct a judgment matrix, and the amount of calculation is reduced by several times compared with the construction of the Analytic Hierarchy Process (AHP) judgment matrix [35].

(1) Determining the order relation.

Experts select the most important indicator in the indicator set $\left\{x_{1}, x_{2}, \cdots, x_{n}\right\}$ and record it as $x_{1}^{*}$. Among the remaining $n-1$ indicators, the most important indicator is selected as $x_{2}^{*}$. By repeating the above steps, after $n-1$ selection, the remaining evaluation indicator is recorded as $x_{n}^{*}$. An order relation $x_{1}^{*}>x_{2}^{*}>\cdots>x_{n}^{*}$ is determined.

(2) Giving a comparative judgement of the importance between $x_{k-1}^{*}$ and $x_{k}^{*}$.

The rational judgment of experts of the ratio $w_{k-1} / w_{k}$ of the importance of the evaluation indicator $x_{k-1}^{*}$ to $x_{k}^{*}$ can be expressed as Formula (3).

$$
w_{k-1} / w_{k}=r_{k}(k=n, n-1, \cdots, 2)
$$

When $n$ is larger, it can be taken as $r_{k}=1 . r_{k}$ can be assigned 1.0,1.2, 1.4, 1.6, or 1.8, depending on the importance of adjacent indicators.

(3) Calculating the weight coefficient $w_{k}$.

According to the rational judgment of $r_{k}$ given by experts, $w_{k}$ is obtained according to Formulas (4) and (5).

$$
\begin{gathered}
w_{k}=\left(1+\sum_{k=2}^{n} \prod_{i=k}^{n} r_{i}\right)^{-1} \\
w_{k-1}=r_{k} w_{k}(k=n, n-1, \cdots, 2)
\end{gathered}
$$

The subjective weight is recorded as $w^{\prime}=\left(w_{1}^{\prime}, w_{2}^{\prime}, \cdots, w_{n}^{\prime}\right)$, which satisfies $0 \leq w^{\prime} \leq 1, \sum_{j=1}^{n} w_{j}^{\prime}=1$.

(2) The objective weight determined by the entropy weight method

The weight obtained by the entropy weight method is based on the degree of variation of indicators, ascertained through information entropy, to get the entropy weight of indicators. The steps used to determine the weight coefficient of the indicators by the method of entropy are as follows [36].

(1) Calculating the contribution degree $p_{i j}$ of scheme $i$ under index $j$.

$$
p_{i j}=x_{i j} / \sum_{i=1}^{m} x_{i j}
$$

(2) Calculating the entropy $e_{j}$ of indicator $j$. 
Entropy $e_{j}$ represents the total contribution of all schemes to index $j$.

$$
e_{j}=-k \sum_{i=1}^{m} p_{i j} \ln p_{i j}
$$

Here, $k=1 / \ln m$, which guarantees $0 \leq e_{j} \leq 1$.

(3) Determining the weight coefficient

The weight coefficient $w_{j}$ is normalized by Formula (8).

$$
w_{j}=\left(1-e_{j}\right) / \sum_{j=1}^{n}\left(1-e_{j}\right)
$$

The objective weight is recorded as $w^{\prime \prime}=\left(w_{1}^{\prime \prime}, w_{2}^{\prime \prime}, \cdots, w_{n}^{\prime}\right)$, which satisfies $0 \leq w^{\prime \prime} \leq 1, \sum_{j=1}^{n} w_{j}^{\prime \prime}=1$.

(3) Determination of the combination weight

In order to reflect the subjective consciousness and objective reality of each indicator in the evaluation, a more reasonable combination of subjective and objective weights is obtained on the basis of the order relation and entropy weight method.

Assuming that $w_{i}^{\prime}$ is the subjective weight of the indicator $i$ determined by the order relationship and $w_{i}^{\prime \prime}$ is the objective weight of the indicator $i$ determined by the entropy weight method, the combined weight of the indicator $i$ is obtained by Formula (9).

$$
w_{i}=\left(w_{i}^{\prime}+w_{i}^{\prime \prime}\right) / \sum_{i=1}^{m}\left(w_{i}^{\prime}+w_{i}^{\prime \prime}\right)
$$

As $\sum_{i=1}^{m}\left(w_{i}^{\prime}+w_{i}^{\prime \prime}\right)=\sum_{i=1}^{m} w^{\prime}+\sum_{i=1}^{m} w^{\prime \prime}=2$, Formula (7) is further deduced into formula (8); that is, the formula for calculating combination weighting.

$$
w_{i}=\left(w_{i}^{\prime}+w_{i}^{\prime \prime}\right) / 2
$$

3.1.2. The Technique for Order Preference by a Similar to Ideal Solution Improved by the Combination Weighting Method

The technique for order preference by a similar to ideal solution is called TOPSIS for short, originating from the discrimination problem in multivariate statistical analysis. With an ideal sample point of $\left(x_{i 1}^{*}, x_{i 2}^{*}, \cdots, x_{i m}^{*}\right)$, the evaluated object $\left(x_{i 1}, x_{i 2}, \cdots, x_{i m}\right)$ needs to be set in the TOPSIS method, and the weighted distance $y_{i}$ between them is defined as follows:

$$
y_{i}=\sum_{j=1}^{m} w_{j} f\left(x_{i j}, x_{j}^{*}\right)(i=1,2, \cdots, n)
$$

where the combination weight coefficients $w_{j}$ are obtained by Formula (10) and the Euclidean distance is usually used for some distance $f\left(x_{i j}, x_{j}^{*}\right)$ between $x_{i j}$ and $x_{j}^{*}$.

Supposing $\left(x_{1}^{+}, x_{2}^{+}, \cdots, x_{m}^{+}\right)$is the positive ideal solution and $\left(x_{1}^{-}, x_{2}^{-}, \cdots, x_{m}^{-}\right)$is a negative ideal solution, the Euclidean distance $y_{i}^{+}$between $\left(x_{i 1}, x_{i 2}, \cdots, x_{i m}\right)$ and positive ideal solution is defined as follows:

$$
y_{i}^{+}=\sum_{j=1}^{m} w_{j} f\left(x_{i j}, x_{j}^{+}\right)(i=1,2, \cdots, n)
$$


The Euclidean distance $y_{i}^{-}$between $\left(x_{i 1}, x_{i 2}, \cdots, x_{i m}\right)$ and the negative ideal solution is defined as follows:

$$
y_{i}^{-}=\sum_{j=1}^{m} w_{j} f\left(x_{i j}, x_{j}^{-}\right)(i=1,2, \cdots, n)
$$

The queuing indicator value $C_{i}$ is shown as Formula (14), which emphasizes the distance from the negative ideal solution. The larger the queuing indicator value, the better the scheme.

$$
C_{i}=\frac{y_{i}^{-}}{y_{i}^{+}+y_{i}^{-}}
$$

\subsection{Support Vector Machine Optimized by an Improved Artificial Bee Colony Algorithm}

With the powerful pattern classification ability of SVM and the excellent global search performance of the ABC algorithm, the energy transition performance evaluation model based on the IABC-SVM is established to solve the problem of a small sample size and complex evaluation process.

\subsubsection{Support Vector Machine}

SVM is a machine learning theory [37]. Firstly, the input space is transformed into a high-dimensional feature space by the non-linear transformation of the kernel function, so that it can be linearly separable, and the optimal classification hyperplane is then obtained in this feature space.

SVM is a machine learning theory based on the principle of structural risk minimization, which was proposed by Corinna Cortes and Vapnik [38] in 1995. It has many unique advantages in a small sample, is non-linear, and can be used for high-dimensional pattern recognition. By searching for the optimal hyperplane to satisfy the classification requirements, the distance between two types of data points and the hyperplane is maximized on the basis of ensuring accurate classification.

For the training sample, $T=\left\{\left(x_{i}, y_{i}\right) \mid i=1,2, \ldots, N\right\}$, where $x_{i} \in R^{d}, y_{i} \in\{+1,-1\}$. The hyperplane is $(\omega \cdot x)+b=0$, and the quadratic programming problem of the optimal classification hyperplane can be shown as follows:

$$
\begin{gathered}
\min \Phi(\omega)=\frac{1}{2}\|\omega\|^{2}+C \sum_{i=1}^{N} \xi_{i} \\
\text { s.t. }(\omega \cdot x)+b \geq 1-\xi_{i}, i=1,2, \ldots, N
\end{gathered}
$$

where $\omega$ is the hyperplane normal vector, $C>0$ is the penalty parameter, $\xi_{i}$ is the relaxation variable, and $b \in R$ is the threshold.

The dual problem of the above quadratic programming problem can be shown as Formula (16).

$$
\begin{gathered}
\max W(\alpha)=\sum_{i=1}^{N} \alpha_{i}-\frac{1}{2} \sum_{i, j=1}^{N} y_{i} y_{j} \alpha_{i} \alpha_{j} K\left(x_{i}, x_{j}\right) \\
\text { s.t. } \sum_{i=1}^{N} \alpha_{i} y_{i}=0,0 \leq \leq C, i=1,2, \ldots, N
\end{gathered}
$$

Here, $\alpha_{i}$ is the Lagrange multiplier and $K\left(x_{i}, x_{j}\right)$ is the kernel function.

The commonly used kernels in SVM are linear kernels, radial basis function kernels, polynomial functions, and sigmoid kernels [39]. In this paper, the radial basis function $K\left(x_{i}, x_{j}\right)=$ $\exp \left(-\left\|x_{i}-x_{j}\right\|^{2} / 2 \sigma^{2}\right)$ is used in SVM. It can be seen that the radial basis function is greatly affected by the parameters $\sigma$. Then, the decision function using the radial basis function is defined as follows:

$$
f(x)=\operatorname{sgn}\left(\sum_{i=1}^{N} \alpha_{i} y_{i} K\left(x_{i}, x\right)+b\right)
$$




\subsubsection{Artificial Bee Colony Algorithm}

The ABC algorithm was proposed by Karaboga D et al in 2005. Compared with other optimization algorithms, the ABC algorithm has a better global search performance [40-42]. Therefore, the algorithm is more frequently applied to the problem of function extremum optimization [43].

The ABC algorithm classifies bee colonies into three categories, including employed bees, onlooker bees, and scout bees. Among them, the employed bee and the onlooker bee with a duty to exploit the honey source account for half of the total bee colony. The role of the scout bee is to avoid too few kinds of honey sources.

When solving optimization problems, the location of the honey source indicates potential possible solutions, and the quality of the honey source indicates the quality of the solution. For the optimization problem, the ABC algorithm randomly generates $N$ honey sources of $D$-dimensional, and employed bees then generate new honey sources according to Formula (18).

$$
v_{i j}=x_{i j}+\operatorname{rand}\left(x_{i j}-x_{k j}\right)
$$

$x_{i j}$ and $v_{i j}$ represent honey sources before and after renewal, respectively. $x_{k j}, k \in\{1,2, \cdots, N\}$ represents randomly selected honey sources, and rand $\in[-1,1]$ is a random number.

The fitness value before and after renewal of the honey source is calculated and greedy selection is conducted to keep better honey sources. The onlooker bee obtains the fitness information from the honey source produced by the employed bee, which is transformed into the solution of the minimization problem.

$$
f_{i t}= \begin{cases}1 /\left(1+f\left(x_{i}\right)\right) & f\left(x_{i}\right)>0 \\ 1+\left|f\left(x_{i}\right)\right| & f\left(x_{i}\right) \leq 0\end{cases}
$$

Here, $f\left(x_{i}\right)$ is the objective function value. The onlooker bee chooses whether to search the honey source in depth according to the probability as Formula (20).

$$
p_{i}=f i t_{i} / \sum_{i=1}^{N / 2} f i t_{i}
$$

Here, $f_{i t}$ is the $i$ th honey source fitness. The greater the fitness, the greater the probability of a deep search.

When one solution is updated a certain number of times, the quality of its solution has not been improved. At this point, the employed bee will be transformed into a scout bee, and a new honey source will be generated by Formula (21).

$$
X_{i j}=\operatorname{rand}(0,1)\left(u_{j}-l_{j}\right)+l_{j}
$$

Here, $u_{j}$ and $l_{j}$ represent the $j$ th-dimension maximum and minimum of the solution obtained in the current cycle, respectively.

\subsubsection{Improved Artificial Bee Colony Algorithm}

Although the $\mathrm{ABC}$ algorithm has a simple structure and excellent performance, it has a strong exploratory ability and insufficient development ability, which makes the convergence speed of the algorithm slow and easy to fall into a local optimum. According to reference [44], the search strategy of the $\mathrm{ABC}$ algorithm in the two stages of the employed bee and onlooker bee is improved.

In the employed bee stage, the honey source component is monitored by introducing a supervisory-response mechanism. In the next iteration process, the state of the supervisor $\varphi$ determines whether the honey source component is updated or not. For example, if the supervisor $\varphi=0$, the last honey source component is updated; otherwise, the honey source component is updated randomly. 
The state of supervisor $\varphi$ depends on whether the quality of the honey source is improved after the component of the honey source is updated in the last iteration. If it is improved, supervisor $\varphi=0$; otherwise, $\varphi=1$. The improved employed bee updating method can improve the development ability of the $\mathrm{ABC}$ algorithm and accelerate the convergence speed of the algorithm.

In the following bee stage, combined with the supervisor of the employed bee renewal process, the probabilistic penalty is imposed on the honey source whose quality has not been improved after the last iteration in the following bee stage. The improved probability formula is as follows:

$$
P_{i}=\frac{1}{1+f_{i}+\varphi_{i} e^{\eta\left(f_{i}-f_{\min }\right)}}
$$

where $P_{i}$ is the honey source probability of the $i$ th employed bee, $f_{i}$ is the fitness value of the $i$ th honey source, $f_{\min }$ is the minimum fitness value, $\eta$ is the probability penalty parameter, and $\varphi_{i}$ represents the state of the supervisor of the $i$ th honey source.

It can be seen that the improved probability formula makes full use of the historical knowledge in the evolutionary process of the employed bee, and punishes the bee sources whose quality is not improved after renewal. It not only enhances the difference of renewal probability among different quality honey sources, but also enhances the co-evolution between the employed bee and onlooker bee, which is conducive to ensuring the further search of excellent bee sources and preventing the algorithm from falling into the local optimum.

3.2.4. Process of the Support Vector Machine Optimized by the Improved Artificial Bee Colony Algorithm

The improved IABC is used to optimize the parameters of SVM to ensure good prediction results. The specific steps are as follows.

The improved $A B C$ algorithm is used to optimize the penalty factor $C$ and radial basis function parameters $\sigma$ of SVM. The steps are as follows:

Step 1. Initialization of parameters. The population number is set to $\mathrm{N}$, the maximum iteration number is set to Max_it, the honey elimination number is set to Max_trial, and the upper and lower limits of the SVM penalty parameter $C$ and radial basis function parameter $\sigma$ are set, generating N/2 honey sources randomly within the range of values. Additionally, the supervisor $S P=\left\{s p_{0}, s p_{1}, \cdots, s p_{N / 2}\right\}$ is initialized;

Step 2. Renewal of employed Bee. Each employed bee randomly selects adjacent honey sources and generates new honey sources. By judging whether the supervisor status continues to update the components of the last honey source, the honey sources before and after the update are judged according to Formula (17).

Taking the classification error rate as the optimization objective, that is the fitness function. The calculation formula is as follows:

$$
E=1-\frac{T}{Q}
$$

where $E$ is the classification error rate, $T$ is the correct number of classifications, and $Q$ is the total number;

Step 3. Renewal of probability selection for onlooker bee. The improved probability selection is used to calculate the honey source probability, and the onlooker bee decides whether to update the honey source further according to the probability selection. If further updates are needed, the update mode of the employed bee in Step 2 is followed;

Step 4. Renewal of the scout bee. If the quality of the honey source does not improve after the employed bee and the onlooker bee have reached the update times of Max_trial, it means that the solution falls into the local optimum and leads the bee to transform into the scout bee to produce a new solution;

Step 5. Determining whether the maximum number of iterations is reached Max_it. If not, go back to Step 2, or end and output the optimal parameters (C, $\sigma)$ of SVM; 
Step 6. The optimized parameter values are assigned to SVM, and the evaluation model is established.

\subsection{Construction of the Performance Evaluation Model of Energy Transition Based on the TOPSIS Method and IABC-SVM}

The basic principle of IABC-SVM for evaluation is that the information used to describe the characteristics of evaluation objects is regarded as the input vector of IABC-SVM and the value of CW-TOPSIS evaluation results is regard as the output of IABC-SVM, and then the model is trained with appropriate samples. Through the IABC algorithm, each iteration has an optimum value of penalty parameter $C$ and radial basis function parameter $\sigma$ suitable for comprehensive evaluation. Then, the IABC algorithm is terminated when the $C$ and $\sigma$ obtained after multiple iterations meet the system error requirement or the number of iterations reaches the maximum. The trained IABC-SVM model can make a good evaluation of similar objects. The evaluation process is shown in Figure 3.

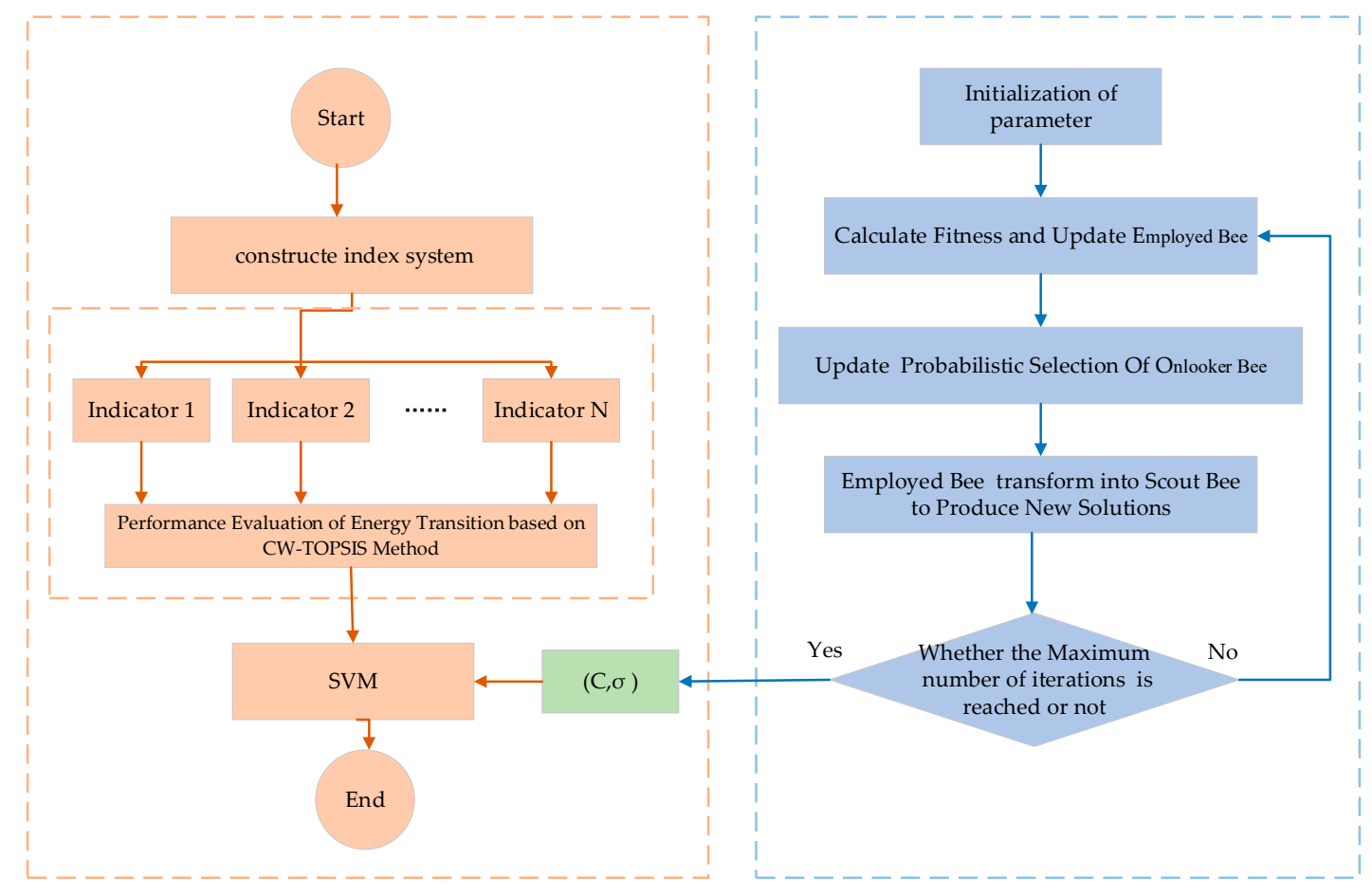

Figure 3. The flow chart of the performance evaluation model of energy transition.

\section{Case Study}

In this section, the energy transition performance of 30 provinces in China is obtained by the CW-TOPSIS method, and are regarded as the expected data of the IABC-SVM model. The validity of the evaluation method has been proved through training and testing. Finally, the energy transition performance of 30 provinces in China is obtained by the IABC-SVM method.

\subsection{Performance Evaluation of Energy Transition Based on TOPSIS}

According to the indicator system of energy transition established in Section 2, the relevant data of 30 provinces in China in 2015 have been collected and preprocessed. The indicators were obtained by processing the available raw data. Among them, the data on GDP, population, R\&D expenditure of industrial enterprises above the scale, industrial waste water, industrial waste gas emissions of major pollutants investment in industrial pollution control, investment in the coal mining and processing industry, and investment in the energy industry were obtained from the China Statistical Yearbook. Data on coal consumption, electricity consumption, energy consumption, power generation, and thermal 
power generation were obtained from the China Energy Statistics Yearbook. The proportion of new energy consumption and the capacity of new photovoltaic installers were obtained from the official website of the Energy Administration.

The evaluation data of qualitative indicators was obtained by inviting 20 experts and scholars, government regulators, and senior managers of power generation enterprises who have a rich knowledge and experience in this field to provide objective and scientific evaluation scores for each qualitative indicator, and taking the average as the final evaluation data of the indicators. Based on the initial values of 18 indicators, the evaluation results were obtained by combination weighting and TOPSIS.

According to the order relation method, 20 experts assigned values according to the ratio of the importance degree to the importance degree of 18 indicators, and the subjective weights of each indicator were then obtained according to Formulas (4) and (5).

$w^{\prime}=(0.0500,0.1200,0.0816,0.1075,0.0380,0.0979,0.0480,0.0567,0.0579,0.0327,0.0472,0.0328$, $0.0394,0.0380,0.0480,0.0328,0.0372,0.0343)$.

According to the entropy weight method, the proportion of each province under one indicator was calculated according to Formula (6), and the entropy weight and objective weight of each indicator were then calculated according to Formulas (7) and (8). When calculating the value of entropy, when the indicator was 0 , the indefinite $\lim _{x \rightarrow 0} x \ln x=0$ appeared because of the operation of the natural logarithm. Then, the objective weights of each indicator were obtained.

$w^{\prime \prime}=(0.0123,0.0606,0.1721,0.0695,0.0138,0.0630,0.0296,0.1422,0.0284,0.0381,0.0681,0.0258$, $0.0604,0.0255,0.0108,0.0457,0.0226,0.1113)$.

Then, the combination weight was obtained according to formula (10):

$w=(0.0312,0.0903,0.1268,0.0885,0.0259,0.0805,0.0388,0.0994,0.0431,0.0354,0.0577,0.0293$, $0.0499,0.0318,0.0294,0.0393,0.0299,0.0728)$.

Finally, the performance of energy transition in each province was obtained based on CW-TOPSIS. According to Formulas (12) and (13), the Euclidean distances between the indicators of each province and the positive and negative ideal solutions were calculated, respectively, and the queuing indicators were calculated according to Formula (14). The energy transition performance and rankings of each province in 2015 were obtained as shown in Table 1.

Table 1. Performance evaluation of energy transition in China in 2015 based on the technique for order preference by a similar to ideal solution improved by a combination weighting (CW-TOPSIS).

\begin{tabular}{cccccc}
\hline Province & Evaluation Value & Ranking & Province & Evaluation Value & Ranking \\
\hline Beijing & 0.999974798 & 1 & Henan & 0.906878532 & 16 \\
Tianjin & 0.994930127 & 4 & Hubei & 0.97923574 & 9 \\
Hebei & 0.706281945 & 23 & Hunan & 0.976428102 & 10 \\
Shanxi & 0.040276805 & 30 & Guangdong & 0.996457913 & 3 \\
Inner Mongolia & 0.421643517 & 25 & Guangxi & 0.952854994 & 14 \\
Liaoning & 0.876709919 & 19 & Hainan & 0.990949087 & 7 \\
Jilin & 0.890888491 & 18 & Chongqing & 0.959191126 & 13 \\
Heilongjiang & 0.804755655 & 22 & Sichuan & 0.974339233 & 11 \\
Shanghai & 0.998218427 & 2 & Guizhou & 0.631780186 & 24 \\
Jiangsu & 0.992414564 & 6 & Yunnan & 0.871834888 & 20 \\
Zhejiang & 0.9935578 & 5 & Shaanxi & 0.833219801 & 21 \\
Anhui & 0.929365368 & 15 & Gansu & 0.406370454 & 26 \\
Fujian & 0.989314852 & 8 & Qinghai & 0.245732554 & 27 \\
Jiangxi & 0.901103627 & 17 & Ningxia & 0.113344232 & 29 \\
Shandong & 0.961389028 & 12 & Xinjiang & 0.182549001 & 28 \\
\hline
\end{tabular}


The higher the score of the evaluation value, the better the energy transition performance, whilst the lower the value, the worse the transition performance. Since the concept of energy revolution was put forward in China at the end of 2014, the performance and ranking of energy transition in 2015 can be taken as a benchmark, and the efforts and development process of energy transition in various provinces can be clearly reflected through the calculation and comparison of energy transformation after 2015.

\subsection{Performance Evaluation of Energy Transition based on IABC-SVM}

\subsubsection{Validation of the Validity of IABC-SVM}

IABC-SVM is trained according to the evaluation results of CW-TOPSIS, where the expected output is the result of the expert evaluation. This paper used 10-fold cross validation, whose purpose of cross-validation is to obtain a reliable and stable model and minimize the prediction error. The data set was divided into 10 parts: nine of them were trained and one was tested in turn.

Finally, the penalty parameter $C$ and the radial basis function parameter $\sigma$ were selected by $N_{R E S S}$, shown as Formula (24), and the parameters that resulted in the minimum value or were no longer smaller were selected as the optimal penalty parameter $C$ and the radial basis function parameter $\sigma$.

$$
N_{\text {RESS }}=\frac{1}{10} \sum_{i=1}^{10}\left(Y_{i}^{\prime}-u\left(X_{i}^{\prime}\right)\right)^{2}
$$

Here, $X^{\prime}, Y^{\prime}$ represent group $i$, and $u\left(X_{i}^{\prime}\right)$ denotes the evaluation made with nine groups of data other than group $i$.

The parameter $(\mathrm{C}, \sigma)$ of SVM was optimized by IABC, and the model parameter settings were as follows. The number $N$ was 60 , the maximum number of iterations $M A X \_I T$ was 100 , the optimization range of penalty parameter $C$ was $[1,500]$, and the optimization range of parameters was $[0.001,200]$.

As shown in Figure 4, when the number of iterations reaches 60 or so, the IABC-SVM model enters a stable state, indicating that the model has been trained at this time. The evaluation results and the residuals are shown in Figure 5 and Table 2.

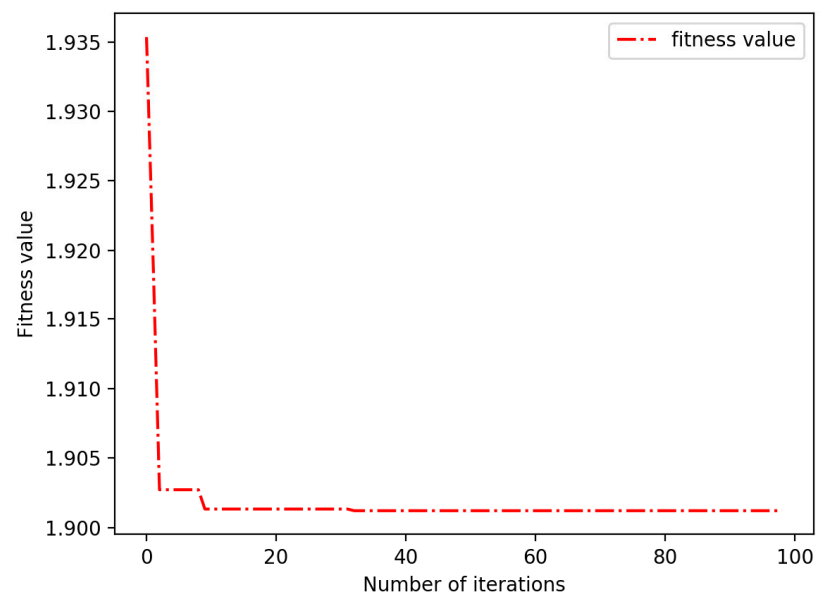

Figure 4. The iterative trend of the the support vector machine improved by the artificial bee colony algorithm (IABC-SVM). 


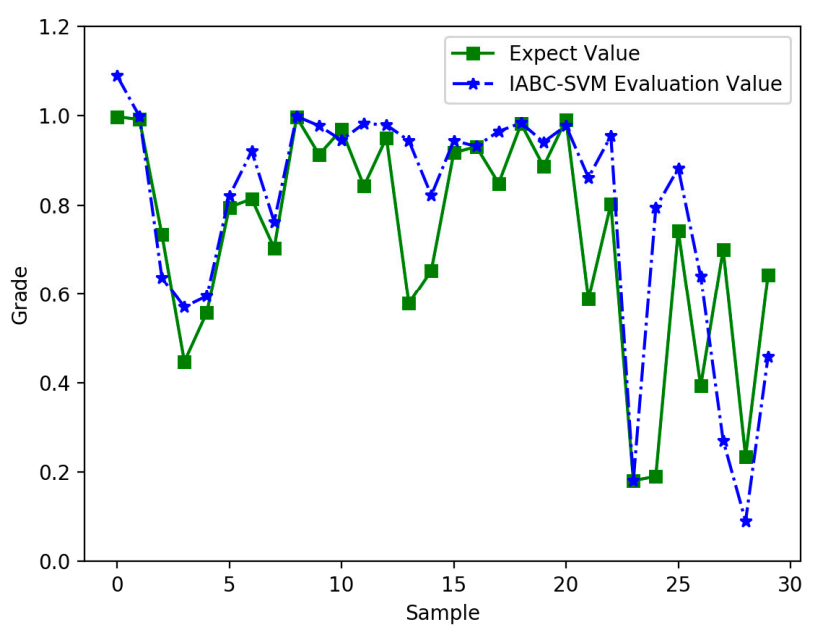

(a)

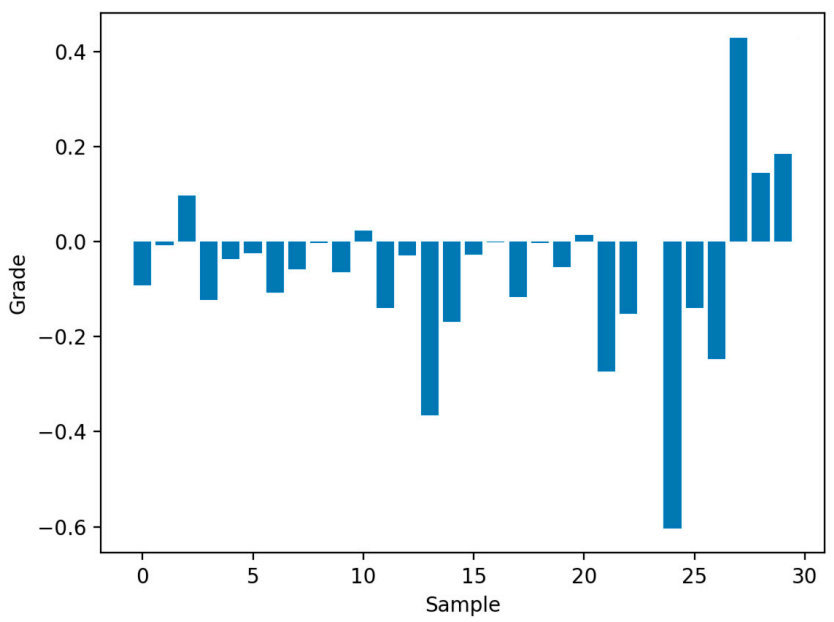

(b)

Figure 5. The evaluation and residual figures obtained by IABC-SVM. (a) The evaluation figure; (b) the residual figure.

Table 2. Performance evaluation of energy transition in China in 2015 based on the support vector machine improved by the artificial bee colony algorithm (IABC-SVM).

\begin{tabular}{cccccc}
\hline Province & Evaluation Value & Ranking & Province & Evaluation Value & Ranking \\
\hline Beijing & 1.029 & 1 & Henan & 0.870147511 & 19 \\
Tianjin & 0.992823783 & 3 & Hubei & 0.925200177 & 12 \\
Hebei & 0.802554534 & 23 & Hunan & 0.99767983 & 2 \\
Shanxi & 0.41800099 & 27 & Guangdong & 0.948266076 & 11 \\
Inner Mongolia & 0.517188298 & 25 & Guangxi & 0.977714263 & 6 \\
Liaoning & 0.813431335 & 22 & Hainan & 0.954606676 & 9 \\
Jilin & 0.914440815 & 15 & Chongqing & 0.92468496 & 13 \\
Heilongjiang & 0.819624016 & 21 & Sichuan & 0.992406785 & 4 \\
Shanghai & 0.991588883 & 5 & Guizhou & 0.133454165 & 29 \\
Jiangsu & 0.921034588 & 14 & Yunnan & 0.886920024 & 18 \\
Zhejiang & 0.912906854 & 16 & Shaanxi & 0.82827724 & 20 \\
Anhui & 0.958763704 & 8 & Gansu & 0.779655892 & 24 \\
Fujian & 0.966572976 & 7 & Qinghai & 0.395541826 & 28 \\
Jiangxi & 0.952641412 & 10 & Ningxia & 0.099 & 30 \\
Shandong & 0.888011509 & 17 & Xinjiang & 0.421211489 & 26 \\
\hline
\end{tabular}


From the evaluation results and errors of test samples, it can be seen that the evaluation results of the IABC-SVM model after training are very close to the traditional evaluation results. It shows that the IABC-SVM constructed in this paper is scientific and reasonable in evaluating the performance of energy transition.

According to Figure 5, IABC-SVM fits the expected results very well when evaluating the effect of energy transition. Furthermore, the errors of IABC-SVM, ABC-SVM, and SVM in evaluating the effect of energy transition are compared and analyzed, including the root mean square error (RMSE), average absolute error (MAE), and average absolute percentage error (MAPE). The comparison results are shown in Table 3 and Figure 6.

Table 3. Error analysis and comparison.

\begin{tabular}{cccc}
\hline Error Types & SVM & ABC-SVM & IABC-SVM \\
\hline RMSE & 0.1211 & 0.0893 & 0.0735 \\
MAE & 0.0769 & 0.0697 & 0.0608 \\
MAPE & 12.6510 & 12.4408 & 11.0894 \\
\hline
\end{tabular}

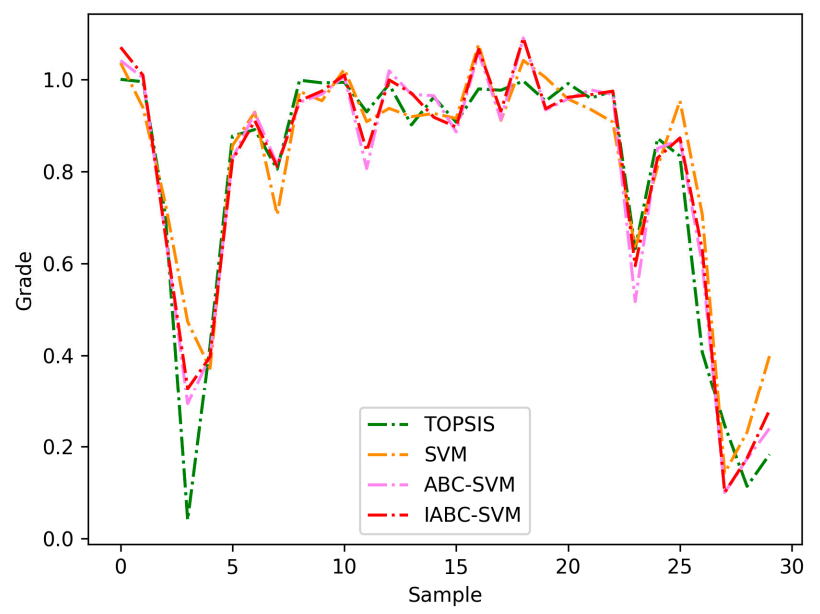

Figure 6. Evaluation results comparison.

From the comparative analysis of the errors in Table 3, we can see that the IABC-SVM evaluation has the smallest errors among the three models. The values of RMSE, MAE, and MAPE are $0.0735 \%$, $0.0608 \%$, and $11.09 \%$ respectively.

From the comparison of the evaluation in Figure 6, it can be seen that the IABC-SVM evaluation model fits the real data well when evaluating the effect of the energy transition. Combining Table 3 and Figure 6, it can be concluded that the order of evaluation accuracy of the three prediction models is IABC-SVM > ABC-SVM > SVM. Therefore, IABC-SVM was selected to evaluate the performance of energy transition of China.

\subsubsection{Performance Evaluation of Energy Transition in 2016 Based on IABC-SVM}

The relevant data of 30 provinces in China in 2016 were collected and pretreated to get the initial values of 18 indicators according to the indicator system. The energy transition performance of the provinces in 2016 was evaluated through the trained IABC-SVM model and compared with that in 2015, as shown in Table 4. 
Table 4. Performance evaluation of energy transition in China in 2016 based on IABC-SVM.

\begin{tabular}{|c|c|c|c|c|}
\hline Province & Evaluation Value & Ranking & Value Changes & Ranking Changes \\
\hline Beijing & 1.0898 & 1 & 0.060778895 & - \\
\hline Tianjin & 0.9987 & 3 & 0.005852718 & - \\
\hline Hebei & 0.6366 & 24 & -0.165993362 & $\downarrow$ \\
\hline Shanxi & 0.5702 & 26 & 0.152228759 & $\uparrow$ \\
\hline Inner Mongolia & 0.5946 & 25 & 0.077456413 & - \\
\hline Liaoning & 0.8202 & 20 & 0.006775219 & $\uparrow$ \\
\hline Jilin & 0.9209 & 16 & 0.006458646 & $\downarrow$ \\
\hline Heilongjiang & 0.7628 & 22 & -0.056843975 & $\downarrow$ \\
\hline Shanghai & 0.9989 & 2 & 0.007299016 & $\uparrow$ \\
\hline Jiangsu & 0.9777 & 8 & 0.056699698 & $\uparrow$ \\
\hline Zhejiang & 0.9455 & 11 & 0.032636907 & $\uparrow$ \\
\hline Anhui & 0.9829 & 5 & 0.024131691 & $\uparrow$ \\
\hline Fujian & 0.9797 & 6 & 0.013148252 & $\uparrow$ \\
\hline Jiangxi & 0.9443 & 13 & -0.008326707 & $\downarrow$ \\
\hline Shandong & 0.8218 & 19 & -0.066197079 & $\downarrow$ \\
\hline Henan & 0.9444 & 12 & 0.074245496 & $\uparrow$ \\
\hline Hubei & 0.9320 & 15 & 0.006774819 & $\downarrow$ \\
\hline Hunan & 0.9652 & 9 & -0.032500267 & $\downarrow$ \\
\hline Guangdong & 0.9842 & 4 & 0.035961433 & $\uparrow$ \\
\hline Guangxi & 0.9411 & 14 & -0.036564819 & $\downarrow$ \\
\hline Hainan & 0.9779 & 7 & 0.023330205 & $\uparrow$ \\
\hline Chongqing & 0.8617 & 18 & -0.063029066 & $\downarrow$ \\
\hline Sichuan & 0.9552 & 10 & -0.037254511 & $\downarrow$ \\
\hline Guizhou & 0.1810 & 29 & 0.047517958 & - \\
\hline Yunnan & 0.7941 & 21 & -0.092859411 & $\downarrow$ \\
\hline Shaanxi & 0.8826 & 17 & 0.054286835 & $\uparrow$ \\
\hline Gansu & 0.6394 & 23 & -0.140206764 & $\uparrow$ \\
\hline Qinghai & 0.2709 & 28 & -0.124642629 & - \\
\hline Ningxia & 0.0902 & 30 & -0.008820512 & - \\
\hline Xinjiang & 0.4585 & 27 & 0.037259839 & $\downarrow$ \\
\hline
\end{tabular}

Several obvious characteristics of energy transformation are found according to the data compared and analyzed in Tables 3 and 4. We divided four types according to the change of transition performance, including the high and stable, remarkable progress, slightly increased, and the lowest of evaluation values and rankings, respectively. Then, we selected typical provinces to analyze their resource endowment, economic development, and energy policies, and find out the advantages and disadvantages of energy transition in typical regions, so as to provide a reference for policy making, as follows:

(1) The evaluation values and rankings of Beijing, Tianjin, Hainan, Shanghai, and Zhejiang province are high and stable. Although the energy structure and mode are different, these provinces have basically entered the stage of diversification, intellectualization, and high-quality development of energy consumption, which can provide reference for other provinces;

(2) The evaluation values and rankings of Anhui, Henan, and Guangdong provinces have made remarkable progress. Anhui province has made some progress in promoting the transformation and development of the coal industry and new energy development. The transition effect of Henan province is closely related to the integration of coal and electricity and the deep processing of coal. Due to the development of wind power and photovoltaic power, and the improvement of natural gas utilization, the transition effect of Guangdong province has been significantly improved. The analysis of provincial transformation measures with an obvious transformation effect can provide an important reference for the energy transition of other provinces;

(3) Shanxi, Inner Mongolia, Shaanxi, and Xinjiang are major energy provinces and key provinces of energy revolution. From the evaluation results, not only the ranking slightly increased, but the score 
has improved. For provinces with a low starting point of transition, we should pay more attention to the score rather than ranking. According to IABC-SVM, the energy transition of energy provinces can be continuously assessed;

(4) Guizhou and Ningxia province have the lowest scores and rankings. Their transformation enthusiasm is not high and the effect is not good. Traditional backward productivity accounts for a large proportion in Guizhou province. Structural contradictions in coal production are prominent, the foundation of scientific and technological innovation is weak, and the market efficiency is low. The resource and environment constraints in Ningxia province are intensifying; the phenomenon of abandoning wind and light is more common; and the task of energy efficiency, energy conservation, and emission reduction is arduous. When formulating energy policy, we should pay more attention to these two provinces.

Compared with the traditional evaluation method, IABC-SVM has obvious differences. IABC-SVM can complete the whole evaluation process independently only by providing the original input data after training. The evaluation process no longer depends on expert scoring and other means, which guarantees the objectivity and scientificity of the comprehensive evaluation process.

In addition, since the penalty parameters $C$ and radial basis function parameters $\sigma$ of IABC-SVM have been determined after the completion of learning, the whole evaluation process can be completed in a very short time, with a high evaluation efficiency, low cost, and better economy.

\section{Conclusions}

In order to simplify the complex process of the traditional evaluation method and evaluate the effect of regional energy transition scientifically and efficiently, this paper has explored the application of the IABC -SVM intelligent evaluation method in the performance evaluation of energy transition. Considering the advantages of the fast convergence of ABC-SVM, the supervisor of the employed bee in the updating process has been introduced, and the probabilistic penalty has been applied to the honey source whose quality is not improved after updating. The IABC-SVM algorithm was obtained to avoid falling into the local optimum. Then, the energy transition performance of 30 provinces in China in 2015 was obtained by the CW-TOPSIS method. Taking the evaluation results as the expected data of the intelligent algorithm, the training and testing of SVM, ABC-SVM, and IABC-SVM prove the effectiveness of IABC-SVM in evaluating the regional energy transition. Then energy transition performance of 30 provinces in China in 2016 was obtained by the IABC-SVM method.

The main contributions of this paper are the evaluation indicator system of energy transition and the intelligent method of energy transition performance evaluation. The establishment of the indicator system is based on the national conditions of China's energy revolution. It is applicable to China's urban energy development assessment after 2014, including provincial analysis and prefecture-level energy transition evaluation. We focused on evaluating the effect of energy transition from the perspective of process by selecting multiple dimensions. In the future, we can focus on the environmental sustainability assessment of energy systems from the perspective of effects, and an indicator system for environmental sustainability assessment will be constructed. The CW-TOPSIS can be used for comprehensive assessment, and relevant data will be used to train the IABC-SVM model, so as to obtain an intelligent evaluation model for environmental sustainability assessment.

The IABC-SVM evaluation method has a good generalization performance in the performance evaluation of energy transition. The transition situation of each year can be rapidly evaluated according to the updated data in the future. Moreover, the indicator system established in this paper is also applicable to the evaluation of the performance of energy transition at the municipal level. This model can provide a good tool for future evaluation of regional energy transition. As long as the initial data are input, the results can be obtained, avoiding organizing expert groups for evaluation and then collecting the presidential accounting process, which can save a lot of time and money, and can simultaneously complete the efficient and accurate evaluation of multiple samples. Through the evaluation results, we can find the problems in energy transition in time and solve them pertinently. 
The applicability of this model is extensive, and it is not limited to evaluating the performance of energy transition. For evaluation problems involving the complex scoring process of experts, this model can be used to learn and realize an intelligent evaluation. However, for evaluation problems that do not involve expert scoring and qualitative evaluation indicators, the learning of this intelligent algorithm will lose its significance.

Author Contributions: All authors have contributed to this paper. Y.L. initiated the project and gave guidance for the methods. Z.L. designed the model and analyzed the data. And Z.L. is responsible for the investigation, resources, and data curation. Y.L. did a great job for the expansion of literature review so as to provide a deeper insight into the related published work and hence establish the contribution of this paper with respect to other publications tracking the problem.

Funding: This paper is supported by the Beijing social science foundation research base project (Grant No. 17JDGLA009), the President fund of China national institute of standardization (Grant No. 572019Y-6774), the Fundamental Research Funds for the Central Universities under No. 2019QN075, and the "Natural Science Foundation of China Project" (Grant No. 71471058).

Acknowledgments: This paper is supported by the Beijing Key Laboratory of New Energy and Low-Carbon Development (North China Electric Power University), Beijing.

Conflicts of Interest: The authors declare no conflicts of interest.

\section{References}

1. Gu, H.B.; Zhang, S. Determination and comparison of energy transformation in China with that in America and Germany. Acad. Res. 2017, 6, 84-91.

2. Liu, L.H.; Zhong, S.M. Energy revolution-Grasp study forth revolution \& one cooperative. Gas Turbine Technol. 2016, 29, 1-8.

3. Cuaresma, J.C.; Hlouskova, J.; Kossmeier, S.; Obersteiner, M. Forecasting electricity spot-prices using linear univariate time-series models. Appl. Energy 2004, 77, 87-106. [CrossRef]

4. Li, H.; Dong, L.; Duan, H.X. On comprehensive evaluation and optimization of renewable energy development in China. Res. Sci. 2011, 33, 431-440.

5. Su, M.; Chen, C.; Yang, Z. Urban energy structure optimization at the sector scale: Considering environmental impact based on life cycle assessment. J. Clean. Prod. 2015, 112, 1464-1474. [CrossRef]

6. Cherp, A.; Jewell, J.; Vinichenko, V.; Bauer, N.; De Cian, E. Global energy security under different climate policies, GDP growth rates and fossil resource availabilities. Clim. Chang. 2013, 136, 83-94. [CrossRef]

7. Loschel, A.; Moslener, U.; Rübbelke, D.T. Indicators of energy security in industrialized countries. Energy Policy 2010, 38, 1665-1671. [CrossRef]

8. Blum, H.; Legey, L.F.L. The challenging economics of energy security: Ensuring energy benefits in support to sustainable development. Energy Econ. 2012, 34, 1982-1989. [CrossRef]

9. Sovacool, B.K.; Mukherjee, I.; Drupady, I.M.; D'Agostino, A.L. Evaluating energy security performance from 1990 to 2010 for eighteen countries. Energy 2011, 36, 5846-5853. [CrossRef]

10. Phdungsilp, A. Assessing energy security performance in Thailand under different scenarios and policy implications. Energy Procedia 2015, 79, 982-987. [CrossRef]

11. Chakravorty, U.; Roumasset, J.; Tse, K. Endogenous substitution among energy resources and global warming. J. Political Econ. 1997, 105, 1201-1234. [CrossRef]

12. Fan, D.C.; Wang, S.H.; Zhang, W. Analysis on the sanity degree of energy structure based on rough set theory. Stat. Inf. Forum 2012, 27, 35-42.

13. Chandler, J. Trendy solutions: Why do states adopt sustainable energy portfolio standards? Energy Policy 2009, 37, 3274-3281. [CrossRef]

14. Dagoumas, A.S.; Barker, T.S. Pathways to a low-carbon economy for the UK with the macro-econometric E3MG model. Energy Policy 2010, 38, 3067-3077. [CrossRef]

15. Han, Z.H.; Sun, Q.L. Comprehensive energy efficiency based on energy, economy and environment subsystems. China Popul. Resour. Environ. 2015, 25, 38-41.

16. Bosseboeuf, D.; Chateau, B.; Lapillonne, B. Cross-country comparison on energy efficiency indicators: The on-going European effort towards a common methodology. Energy Policy 2007, 25, 673-682. [CrossRef] 
17. Herrerias, M.J. World energy intensity convergence revisited: A weighted distribution dynamics approach. Energy Policy 2012, 49, 383-399. [CrossRef]

18. Duro, J.A.; Padilla, E. Inequality across countries in energy intensities: An analysis of the role of energy transformation and final energy consumption. Energy Econ. 2011, 33, 474-479. [CrossRef]

19. Zhu, C.; Shi, Z.B. Study of environmental performance assessment of key energy transformation policies. Environ. Impact Assess. 2017, 39, 4-9.

20. Ziemba, P. Inter-criteria dependencies-based decision support in the sustainable wind energy management. Energies 2019, 12, 749. [CrossRef]

21. Wang, C.-N.; Yang, C.-Y.; Cheng, H.-C. Fuzzy multi-criteria decision-making model for supplier evaluation and selection in a wind power plant project. Mathematics 2019, 7, 417. [CrossRef]

22. Wang, C.-N.; Nguyen, V.T.; Thai, H.T.N.; Tran, N.N.; Tran, T.L.A. Sustainable supplier selection process in edible oil production by a hybrid fuzzy analytical hierarchy process and green data envelopment Analysis for the SMEs food processing industry. Mathematics 2018, 6, 302. [CrossRef]

23. Zhao, Y.; Xiang, J.; Xu, J.; Li, J.; Zhang, N. Study on the comprehensive benefit evaluation of transnational power networking projects based on multi-project stakeholder perspectives. Energies 2019, 12, 249. [CrossRef]

24. Dinmohammadi, A.; Shafiee, M. Determination of the most suitable technology transfer strategy for Wind turbines using an integrated AHP-TOPSIS decision model. Energies 2017, 10, 642. [CrossRef]

25. Wu, J.W.; Liang, X.Y.; Yu, B.; Jin, J.; Wang, D.W. Research on the application of BP artificial neural network in DSM comprehensive evaluation. Intell. Build. 2016, 11, 51-55.

26. Niu, D.; Li, Y.; Dai, S.; Kang, H.; Hue, Z.; Jin, X.; Song, Y. Sustainability evaluation of power grid construction projects using improved TOPSIS and least square support vector machine with modified fly optimization algorithm. Sustainability 2018, 10, 231. [CrossRef]

27. Niu, D.; Li, S.; Dai, S. Comprehensive evaluation for operating efficiency of electricity retail companies based on the improved TOPSIS method and LSSVM optimized by modified ant colony algorithm from the view of sustainable development. Sustainability 2018, 10, 860. [CrossRef]

28. Liu, N. Study on the Effect of Fixed Assets Investment in the Energy Industry on Carbon Dioxide Emissions. Master's Thesis, China University of Mining and Technology, Beijing, China, 2016.

29. Zhong, P.F. Application of quality management in centralized purchasing management of coal-fired power plants. Intern. Combust. Engine Parts 2018, 01, 174-180.

30. Xiong, Y.; Liu, Y. Pollution treatment evaluation research on Beijing-Tianjin-Hebei region and the Yangtze river delta city group—Based on PSR evaluation system. China Dev. 2018, 18, 13-18.

31. Wang, Y.Q. The factor analysis method and its application of energy consumption intensity variation. J. Quant. Tech. Econ. 2003, 08, 151-154.

32. Chen, Y.; He, D.F. The difference in provincial R\&D expenditure and the relation of this expenditure with GDP per capita. China Soft Sci. 2012, 10, 78-87.

33. Laurent, A.; Owsianiak, M. Potentials and limitations of footprints for gauging environmental sustainability. Curr. Opin. Environ. Sustain. 2017, 25, 20-27. [CrossRef]

34. Kang, L.X. Research on Industrial Policy and Transformation of Local Government in China. Doctor's Thesis, Capital University of Economics and Business, Beijing, China, 2014.

35. Wang, X.J.; Guo, Y.J.; Lan, T. Rank correlation analysis of formation of consistent judgment matrix. J. Northeast. Univ. (Nat. Sci.) 2006, 27, 115-118.

36. Qin, Z.C.; Chen, G.B.; Li, T.; Sun, W.; Fu, B. CW-TOPSIS mine internal caused fire evaluation model of "AHP + entropy weight method". J. Xi'an Univ. Sci. Tech. 2018, 38, 193-201.

37. He, Y.X. Comprehensive Evaluation Method and Application of Electric Power; China Electric Power Press: Beijing, China, 2011.

38. Vapnik, V. The Nature of Statistical Learning Theory; Springer: Berlin, Germany, 1995.

39. Zhou, S.L.; Liao, J.; Shi, X.J. SVM parameters selection method based on Fisher criterion and maximum entropy principle. Control Decis. 2014, 29, 1991-1996.

40. Karaboga, D.; Basturk, B. A powerful and efficient algorithm for numerical function optimization: Artificial bee colony (ABC) algorithm. J. Glob. Optim. 2007, 39, 459-471. [CrossRef]

41. Karaboga, D.; Akay, B. A comparative study of artificial bee colony algorithm. Appl. Math. Comput. 2009, 214, 108-132. [CrossRef] 
42. Jiang, B.S.; Liu, S.; Kang, Y.X. Research on investment risk evaluation of general aviation enterprises based on ABC-SVM. Friends Account. 2018, 09, 106-112.

43. Xingbao, L.; Zixing, C. Artificial bee colony programming made faster. In Proceedings of the Fifth International Conference on Natural Computation IEEE, Tianjin, China, 14-16 August 2009; pp. 154-158.

44. Wang, D.D.; Zu, Y.; Zhu, P. Advanced artificial bee colony algorithm for SVM optimization and its application on sentiment classification of product reviews. Comput. Appl. Softw. 2017, 34, 33-37.

(C) 2019 by the authors. Licensee MDPI, Basel, Switzerland. This article is an open access article distributed under the terms and conditions of the Creative Commons Attribution (CC BY) license (http://creativecommons.org/licenses/by/4.0/). 